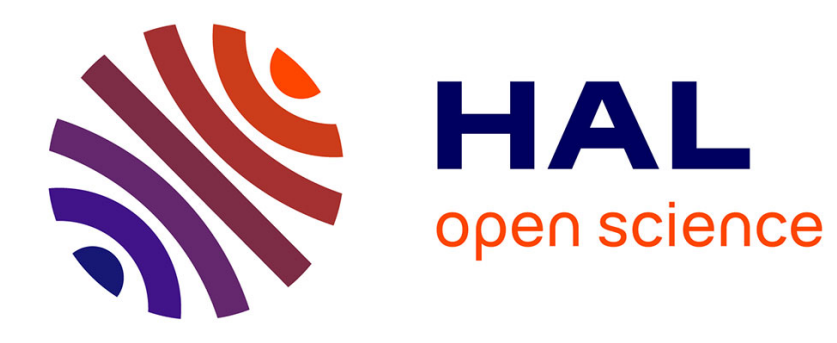

\title{
Quand ethnologue et imam croisent leurs plumes
}

\author{
Marie Miran-Guyon
}

\section{To cite this version:}

Marie Miran-Guyon. Quand ethnologue et imam croisent leurs plumes: Récit d'un voyage au pays de l'anthropologie collaborative. Cahiers d'études africaines, 2010, 198-199-200, pp.951-980. halshs01406177

\section{HAL Id: halshs-01406177 https://shs.hal.science/halshs-01406177}

Submitted on 30 Nov 2016

HAL is a multi-disciplinary open access archive for the deposit and dissemination of scientific research documents, whether they are published or not. The documents may come from teaching and research institutions in France or abroad, or from public or private research centers.
L'archive ouverte pluridisciplinaire HAL, est destinée au dépôt et à la diffusion de documents scientifiques de niveau recherche, publiés ou non, émanant des établissements d'enseignement et de recherche français ou étrangers, des laboratoires publics ou privés. 


\title{
Quand ethnologue et imam croisent leurs plumes
}

\author{
Récit d'un voyage \\ au pays de l'anthropologie collaborative ${ }^{1}$
}

\begin{abstract}
« Je ne crois pas aux constatations objectives. Faisceau infini d'interactions humaines » (Hillesum 1995). « La connaissance progresse en intégrant en elle l'incertitude, non en l'exorcisant $\gg$ (Morin 2008).
\end{abstract}

Dans leur livraison 195 de 2009, les Cahiers d'Études africaines ont publié un long article intitulé «(Auto)biographie d'une conversion à l'islam : Regards croisés sur une histoire de changement religieux dans le Bénin contemporain », que je co-signais avec El Hadj Akan Charif Vissoh, le converti en question devenu imam de la mosquée centrale d'Allada. Le présent article retrace la généalogie de cette expérience singulière, à la fois relation intersubjective et coproduction intellectuelle, à tous égards inspirante et fructueuse pour les deux interlocuteurs que nous fûmes amenés à devenir et que nous sommes restés. Par défaut et avec une pointe de regret cependant, il éclaire davantage mon point de vue que celui de Vissoh, que je sollicitais sur ce terrain d'échange réflexif mais sans imposer ce qui parut peut-être relever - non sans fondement ! - de ratiocinations idiosyncratiques universitaires (nous caressons en revanche le projet d'une autre collaboration en terrain musulman béninois). Cet article sacrifie ainsi au rite du récit ethnographique à la première personne ou " confessional tale » pour reprendre l'expression mordante de Van Maanen (1988; Okely \& Callaway 1992).

1. Mes chaleureux remerciements à Robert Launay, André Mary et surtout JeanPierre Olivier de Sardan pour la générosité de leurs commentaires critiques et de leurs conseils de lecture. Si l'article y a gagné en nuances, ses failles restent miennes. Les propos non référencés de ces collègues dans mon texte font directement écho à ces commentaires (pour surenchérir dans l'exercice pratique d'écriture polyphonique!)

Cahiers d'Études africaines, L (2-3-4), 198-199-200, 2010, pp. 951-980. 
Bien qu'endossant un ton délibérément personnel et introspectif, j'espère néanmoins circonscrire certains excès narcissiques propres à l'exercice - l'anthropologue se propulsant aux devants de la scène et exhibant l'inventaire de sa bibliothèque (Spencer 1989) — et ce, en privilégiant une posture pragmatique et (auto)critique, préoccupée par les conditions de validation et les limites inhérentes à ce genre d'aventure collaborative.

El Hadj Akan Charif Vissoh, né Akan Lucien Vissoh en 1953 en pays mahi près d'Abomey, est une personnalité composite et cosmopolite, autant par amour des langues (outre le fon, il parle couramment le français, l'anglais et l'arabe), par expérience biographique (il vécut quatorze ans en Côte-d'Ivoire) que par l'ouverture d'esprit caractéristique de son « être au monde ». Passeur de frontières, Vissoh l'a été aussi au plan religieux. En dépassant la culture clanique vodun associée à sa mère et l'héritage catholique transmis par son père, il fit le pari d'entrer dans l'islam en 1974 avant de devenir, lentement, au fil d'années sombres et lumineuses, par la conjugaison d'un apprentissage des dogmes religieux et de la pratique sociale des rites islamiques, le musulman qu'il est resté, hier simple fidèle, désormais intellectuel et chef spirituel musulman. Chevauchant plusieurs mondes, Vissoh se fit traducteur, non seulement parce qu'il entreprit de traduire le Coran de l'arabe en langue fon, mais surtout parce qu'il n'eut de cesse de traduire diverses réalités socioreligieuses à divers publics de croyants, en appelant les musulmans nés dans l'islam mais aussi les nonmusulmans à troquer le rideau de leurs préjugés réciproques pour l'horizon d'une plus grande compréhension et respect mutuels. À la force du poignet, Vissoh s'est inventé un destin d'éducateur par excellence. Sans être un double de l'ethnologue, il a été un partenaire hors pair, auprès de qui il me fut donné de m'abreuver, telle à une source vive et fraîche, à de nouvelles connaissances et modes de connaissances.

Je chérissais pour ma part un même cosmopolitisme éthique et pratique, ayant vécu dans des milieux laïcs baignés de cultures religieuses catholique, protestante et juive sur trois continents, et pour près de quinze ans entre Israël, l'Angleterre et les États-Unis. Je reçus une formation doctorale en histoire de l'Afrique et du Moyen-Orient et je fus ensuite recrutée pour enseigner l'histoire de l'Afrique dans des départements d'histoire, notamment à la School of Oriental and African Studies (SOAS) de Londres. De manière symbolique ou anodine, ma collaboration avec Vissoh correspondit cependant à une sorte de conversion professionnelle vers l'anthropologie, qui devint ma discipline de référence lorsque je rejoignis Paris et l'École des hautes études en sciences sociales (EHESS). La pratique vénérée de la multidisciplinarité invalide sans doute l'idée même de conversion de champ scientifique. Mieux : c'est une position de principe à laquelle je suis résolument attachée. Mais on ne peut esquiver complètement la réalité de nos institutions d'éducation supérieure, que le contexte national teinte aussi de nuances spécifiques. La gestion universitaire relative aux formations des étudiants, aux acquisitions pour les bibliothèques, aux publications dans des 
revues scientifiques, aux grands congrès (surtout en pays anglo-saxon), aux sources de financement de la recherche ou aux règles de promotion pour le corps professoral : cette gestion, donc, est cloisonnée et jalouse de ses prérogatives autant que poreuse et interdisciplinaire. Sans être transgression, le passage d'une approche de la recherche plutôt nourrie par l'histoire à une démarche davantage inspirée par l'anthropologie n'est pas qu'anecdotique. Il a même des aspects concrets, dont ce rite de passage - initiatique ? par un exercice réflexif sur mon rapport au terrain!

Le fait est que cet article n'est pas le fruit d'une initiative spontanée mais celui d'une sollicitation amicale de communication orale ${ }^{2}$. Cette invitation me permit d'entreprendre une plongée au long cours dans un océan de lectures référentielles inspirantes qui m'étaient pour partie nouvelles, provoquant en retour une prise de conscience fort saine de ma naïveté initiale dans mon rapport à Vissoh - et accessoirement un étonnement pouvant friser l'incrédulité devant la virulence des tempêtes que les anthropologues de tous bords déchaînent parfois dans des tasses de thé, avec des conséquences ambiguës quant à l'avancement de notre compréhension collective des réalités prioritaires du terrain. Cet article révisé prend prétexte de l'exposé singulier de ma collaboration avec Vissoh pour esquisser un voyage de modeste encablure dans l'histoire et l'épistémologie de l'anthropologie, en favorisant les aspects pratiques sur les aspects théoriques, sans oublier ni délégitimer la multidisciplinarité. Loin de moi, cependant, l'ambition de brosser un portrait encyclopédique des transmutations intervenues ces cent cinquante dernières années d'une anthropologie occidentalo-centrée issue des Lumières à une anthropologie postmoderne de la contemporanéité globalisée et multiculturelle, où prévaut la «co-temporalité » des chercheurs et de leurs informateurs, comme l'écrit pertinemment Fabian (1983) : la littérature métaanthropologique - surtout en langue anglaise mais aussi en français — est d'une richesse débordante sur cette trajectoire faite de tournants successifs qui épousent les évolutions du siècle (Asad 1990 ; Bensa 2006 ; Bensa \& Fassin 2008 ; Cefaï 2010 ; Clifford 1983a ; Clifford \& Marcus 1986 ; Jackson 1998 ; Marcus 1998 ; Marcus \& Fisher 1986). En novice, mon intention n'est que d'inscrire ma démarche de co-écriture dans la perspective d'un courant ancien quoique méconnu car longtemps marginalisé au sein de la discipline, celui de l'ethnographie collaborative. En fait, ce courant semble désormais gagner du terrain et des lettres de noblesse comme l'attestent la création en 2006 d'une chaire en "anthropologie collaborative » au sein du département d'anthropologie sociale de l'Université de Cambridge ${ }^{3}$, la parution en 2005 du Chicago Guide to Collaborative Ethnography d'Eric Lassiter (2005b), également

2. Mes remerciements à Éloi Ficquet pour son invitation à prendre la parole lors de la séance inaugurale des « Débats de l'EHESS » sur « l'actualité du terrain », Paris, 22-24 octobre 2007.

3. Caroline Humphrey est la première "Sigrid Rausing Professor of Collaborative Anthropology» au King's College de l'Université de Cambridge. 
directeur de publication de la revue Collaborative Anthropologies lancée en 2008 aux presses de l'Université du Nebraska ${ }^{4}$.

L'anthropologie collaborative, qui valorise (sans se résumer à) la coproduction et la co-signature de textes avec des acteurs non professionnels issus des communautés étudiées ${ }^{5}$, n'est pas sans rappeler la démarche de l'anthropologie réciproque (Bouju 2003). À la différence cependant des beaux projets en anthropologie réciproque tels que l'entreprise monumentale de Transcultura (Le Pichon 1991), l'ethnographie collaborative ne fait se croiser les regards du chercheur et de l'acteur partenaire (ou multiplicité d'acteurs) que dans une perspective dialogique outsider-insider autour de l'acteur et de sa communauté, sans impliquer en retour un projet cognitif équivalent sur le chercheur lui-même en tant que sujet individuel et social, quand bien même le terrain d'enquête se trouve multisitué. C'est là l'une de ses limitations liminaires.

Outre-Atlantique, les nouveaux chantres de l'ethnographie collaborative $\mathrm{y}$ voient volontiers une panacée aux problèmes éthiques qui ont longtemps nourri la mauvaise conscience des praticiens occidentaux de la discipline. Pour certains, le tournant collaboratif irait jusqu'à fonder une nouvelle « anthropologie publique » consciente de l'exigence scientifique autant que du devoir moral de prêter l'oreille aux questions et aux besoins exprimés par les communautés de base elles-mêmes, en y articulant dès lors ses projets from the ground up, aux antipodes de l'approche hiérarchique top-down (Field 2008b ; Fluehr-Lobban 1990, 2008 ; Lassiter 2005 ; Rappaport 2008). La pertinence du débat est évidente. L'esquisse de réponses est généreuse et gonflée de promesses. Mais la ferveur quelque peu messianique ${ }^{6}$ de la défense de cet engagement anthropologique n'est pas sans poser problème, notamment en ce qu'elle esquive l'épineuse question de l'activisme militant dans les sciences humaines (D’Andrade \& Scheper-Hughes 1995 ; Cefaï \& Amiraux 1992), mais aussi parce qu'elle balaye trop rapidement voire dénigre indûment d'autres manières de penser, de faire et d'écrire ${ }^{7}$.

4. Voir <http://www.marshall.edu/coll-anth/>.

5. L'ethnologue est le plus souvent exogène au groupe étudié. Le contraire est un cas particulier, qui interroge l'intériorisation du croisement des regards (voir par exemple RANCO 2006) mais n'exclut pas la possibilité de malentendus voire de tensions entre la communauté étudiée et son interprète endogène (pour un exemple en contexte musulman, voir KASSAM 2004). Un autre cas particulier est la collaboration entre ethnologues «professionnels » de différentes origines géographiques ou culturelles (un exemple est AYI, HARRELL \& LUNZI 2007).

6. Cette ferveur est typique d'une certaine «righteousness » nord-américaine qui n'épargne pas les milieux universitaires et est d'autant plus prononcée qu'elle s'inscrit dans un marché farouchement compétitif de l'offre et de la demande des idées (par ailleurs incomparablement stimulant). De la capacité à «percer» dans ce marché dépend aussi la sécurité de l'emploi et la promotion des enseignants-chercheurs, bien plus directement qu'en Europe.

7. Sur ces débats en France, voir par exemple LESERVOISIER et VIDAL (2007), BIERSCHENK (2007) sur l'œuvre d'Olivier de Sardan et Olivier DE SARDAN (2008). 
Aussi me rangerais-je prudemment sur ce point du côté de celles et de ceux qui considèrent que la coexistence de modalités plurielles de rapports au terrain et d'écriture ethnographiques est une richesse, reflet de nos diverses personnalités et sensibilités de recherche tout autant que de l'indépassable complexité de notre monde, face à la compréhension duquel s'impose à nous une bonne dose d'humilité, comme y invitent les citations placées en exergue. Je m'y soumets d'autant plus volontiers que mon expérience de co-écriture avec Vissoh s'est construite sur le tas, à tâtons, dans un mélange d'intuition, de considérations éthiques et de bricolages pragmatiques. De même, ma réflexion subséquente sur la pratique peu conventionnelle si ce n'est hétérodoxe du brouillage des cartes entre l'interprète et l'interprété, sur le rapprochement entre le terrain comme siège du relationnel et l'écriture comme distanciation a-sociale (contre la tradition malinowskienne : Mosse [2006]) et sur le glissement de l'idée de restitution à celle de collaboration transculturelle (Augé 1994) : ma réflexion, donc, n'a pas non plus la solidité de la maturité. Elle ne vise pas à faire autorité. Comme me le faisait remarquer André Mary, elle relève plutôt d'une prise de risque. Elle est en phase d'exploration cognitive, portée par une croyance débordant peut-être la rationalité quant à la possible fécondité d'une telle collaboration, qui mérite alors d'être exposée à la (ré)évaluation critique des pairs, dans l'espoir — qui sait ? - de nouer de nouveaux dialogues.

Bref retour sur les études africaines et sur les études sur l'islam subsaharien

Quelques remarques sur le contexte plus large dans lequel s'est inscrite ma collaboration avec Vissoh peuvent en éclairer ses sources d'influence et ses étapes. Il y a d'abord le contexte des études africaines. Exception faite peutêtre du cas de l'Éthiopie et de l'Afrique du Sud, c'est un fait que les chercheurs et les institutions basés en Europe et en Amérique du Nord, toutes disciplines confondues, dominent très largement la production du savoir scientifique sur l'Afrique subsaharienne. Entre autres nombreuses voix, celle du philosophe béninois Paulin Hountondji (1990, 1995) s'est élevée contre la dure réalité de cette autre inégalité de développement. De fait, à l'instar de l'orientalisme, les études africaines s'apparentent à l'origine à une invention européenne ayant partie liée à l'entreprise de subjugation et de colonisation du continent (Mudimbe 1988; Amselle 1999). Si l'on ne peut engoncer l'ensemble des anthropologues de l'époque coloniale en un prototype caricatural, reste qu'il domine de leur littérature collective un déni d'histoire et un déni de capacité d'initiative ou de changement, réduisant l'Afrique et ses habitants à un éternel présent primitif : trope des critiques postcoloniales et subalternes. L'anthropologue, s'affublant d'objectivité distancée, s'attribuait alors la légitimité exclusive à décrire tous les aspects de ce présent indifférencié, ce que d'aucuns qualifièrent ensuite de «pillage » du savoir 
des sociétés africaines. Qui plus est, ce savoir s'écrivait généralement de manière surplombante «par dessus l'épaule des indigènes » (Lassiter 2005b), c'est-à-dire dans le déni de la médiation d'intermédiaires locaux, rarement mentionnés et encore moins remerciés ${ }^{8}$. Les rares intellectuels africains à produire un savoir alternatif, principalement dans les colonies britanniques, n'étaient guère écoutés (Sanjek 1993).

À rebrousse-poil, dans la lignée de l'initiative pionnière de l'Institut Rhodes-Livingstone lancée autour de la Seconde Guerre mondiale en Rhodésie du Nord par des anthropologues sociaux britanniques épousant la cause de la gauche (Schumaker 2001), les études africaines sont ensuite devenues très largement anti-coloniales et anti-hégémoniques, au point qu'à la différence peut-être d'autres aires culturelles, l'engagement de l'africaniste occidental pour son sujet dépasse souvent le seul niveau professionnel. Une université à fort ancrage africaniste telle que Michigan State University a, par exemple, joué un rôle pionnier dans le boycott américain contre l'Afrique du Sud du temps de l'apartheid ${ }^{9}$. À la différence des études sur le Moyen-Orient (Kramer 2001), passée l'ère des indépendances, les études africaines, notoirement aux États-Unis, ont massivement tourné le dos aux financements publics dont les projets véhiculaient des préoccupations d'ordre sécuritaire pour les pays du Nord. L'empathie et même la sympathie pour le terrain d'étude sont devenues une réalité partagée par la majorité des spécialistes de l'Afrique noire, au point de poser le problème de l'autocensure face à des sujets délicats tels que l'esclavage interne africain dit «moderne» (Botte 2000), sous-tendu par la crainte de conforter l'image déjà négative que se forme une certaine opinion publique occidentale de l'Afrique contemporaine.

Le problème, qu'il serait indécent et préjudiciable de nier, c'est que cette empathie ne résout pas le problème structurel d'une certaine dépendance intellectuelle entre Afrique et Occident, relatif à la violence symbolique qui existe dans l'appropriation du vécu et de la mémoire d'individus et de sociétés qui n'ont pas toujours les moyens de s'exprimer et de se faire entendre en retour. Le débat dépasse de loin les polémiques sur l'afrocentrisme et l'afrocentricité, au demeurant peu acerbes en France. Qui décide de ce qu'il est important d'étudier sur l'Afrique subsaharienne ? Quels projets doivent être financés et publiés, selon quelle conception du savoir, et pour quel public ? À cet égard : point de bilan facile et transparent. Comme ont pu l'écrire non sans justesse Owusu (1976), Fabian (1983, 1990, 2006), McGrane (1989) ou van Dijk \& Pels (1996) parmi d'autres, même les tendances les plus postmodernes, critiques, réflexives ou littéraires de l'anthropologie ne se sont pas toujours affranchies d'un «impérialisme cognitif

8. Pour un examen critique des pratiques de terrain de Monica Hunter dans la région du Cap dans les années 1930, voir BANK (2008).

9. Voir <http://africanactivist.msu.edu/>. 
extrêmement subtil », dans la mesure où la discipline reste " plutôt un monologue analytique sur les autres cultures que le résultat d'un dialogue avec elles » (Ghasarian 1997 : 192, reprenant McGrane). L'anthropologue ougandaise Christine Obbo (1990) déplore ainsi la persistance d'une inégalité professionnelle face à ses homologues des pays du Nord, lesquels ne seraient pas toujours bien disposés en outre à reconnaître pleinement la contribution de leurs collaborateurs locaux (j'ai plus d'une fois entendu cette même plainte de la bouche de chercheurs basés en Côte-d'Ivoire).

Ces questions lancinantes ont encore refait surface en juin 2007 sur la liste de discussion sur Internet $\mathrm{H}$-Africa ${ }^{10}$, sorte d'espace public savant, transnational et pluridisciplinaire, animé par le milieu universitaire travaillant sur l'Afrique. Elles y sont abordées lucidement dans un message de l'historien de l'Afrique Jonathan Reynolds, reprenant l'introduction de sa communication au «Symposium sur le terrain en Afrique » qu' avait organisé la West African Research Association (WARA) à Dakar en 2002. Son titre seul en dit long: « Mining Information and Manufacturing Scholarship. Are Western-Based Researchers Just Another Extractive Economy? » Reynolds s'inquiète de la relégation de l'Afrique au rang de simple pourvoyeuse de matières premières, non seulement dans le domaine de la production industrielle aux mains de multinationales étrangères que les chercheurs basés en Occident aiment à détester, mais aussi de la production du savoir intellectuel sur le continent que ces mêmes chercheurs tendent à contrôler. La mise en parallèle est troublante. Comme dans l'économie politique du secteur privé, le chercheur basé en Occident a besoin que des institutions dotées en capital investissent dans ses recherches. Il utilise ces financements pour accumuler du data brut lors de ses terrains, facilités par le moindre coût du travail en Afrique. Les matériaux une fois «extraits », le chercheur se replie sur son institution en Europe ou en Amérique du Nord pour les « raffiner», les « vendre » à des maisons de publication, et assurer par suite la progression de sa carrière et de son salaire. Sur un ton autocritique pesé que j'endosse volontiers, Reynolds s'interroge alors sur les moyens à même de rendre moins extractifs les mécanismes du métier, pour le mettre en adéquation avec les idéaux éthiques dont il se croit porteur ${ }^{11}$. Ces débats éclairent concrètement l'acuité des défis relatifs à l'échange avec les interlocuteurs et les sociétés subsahariens, à la restitution du texte scientifique et à la capacité d'écoute à sa réception locale ${ }^{12}$.

10. Voir <http://www.h-net.org/ africa/>.

11. Reynolds sur H-Africa Discussion Logs, 27 Juin 2007.

12. Launay me fait remarquer à juste titre que les sociologues et autres chercheurs en sciences humaines ne travaillent pas que sur les sociétés en voie de développement: les pratiques en terrain européen ne sont pas moins « extractives ». La question de l'asymétrie de pouvoir n'a donc rien de spécifique aux situations coloniales et néocoloniales (elle est même virtuellement intrinsèque à la production de tout discours savant), même si elle est plus criante dans le cas de ces dernières. Launay invite aussi à ne pas confondre la marginalisation des universitaires africains (dont les projets et les objectifs peuvent être comparables aux 
Il y a ensuite le contexte des études sur l'islam en Afrique subsaharienne. Pendant la période coloniale, tout particulièrement du côté français, ce champ d'études fut investi par des administrateurs coloniaux et des hommes d'Église formés dans le souci de surveiller les musulmans ${ }^{13}$. Puis il évolua jusque dans les années 1970 en un sous-domaine érudit, dominé par des historiens arabisants, à la périphérie des études africaines et plus encore des études islamiques - ce qui explique d'ailleurs qu'il n'ait guère été touché par les controverses sur l'orientalisme. La mondialisation de la résurgence islamique a profondément changé la donne en renouvelant les questionnements autant que le personnel de recherche, élargi aux anthropologues et aux politologues ${ }^{14}$. Dans le même temps, les centres de recherche et les universités islamiques se sont aussi multipliés dans les pays africains à majorité musulmane. Mais parce que la littérature produite par leurs intellectuels non europhones - pour reprendre l'expression générique d'Ousmane Kane (2003) - propageait une vision normative de l'islam, elle n'était pas considérée en Occident comme académique : tout au plus comme une source primaire (Hodgkin 1990). Il n'y avait donc pas de dialogue entre ces deux formes de savoir : le premier exogène, séculier, critique ; le second endogène, religieux, apologétique.

nôtres) et la situation de nos collaborateurs non universitaires (dont les agendas et les priorités peuvent être très différents). La marginalisation des universitaires africains n'a pas que partie liée aux postures de leurs collègues euraméricains. Elle résulte tout autant, si ce n'est plus, d'un vaste faisceau de circonstances, au premier chef desquelles la marginalisation économique de leur pays d'origine et le manque de ressources de leurs universités. Cela ne délégitime en rien le souci des chercheurs du Nord à l'endroit des conditions d'exercice du métier de leurs collègues du Sud, mais en relativise un tant soit peu la portée : il ne faudrait pas verser dans l'excès de tout ramener indûment à soi, surtout si l'enjeu n'est que de conforter sa bonne conscience... Olivier de Sardan m'invite pour sa part à désagréger soigneusement les divers niveaux de relations, de registres et d'échelles qui sont en jeu dans la dénonciation d'une « antropologie extractive ». Le niveau des relations inter-individuelles, relativement idiosyncratiques, n'est pas celui des différents contextes institutionnels ni celui des comptes non réglés de la colonisation. Olivier de Sardan en appelle finalement à ne pas négliger l'importance de la question de la citoyenneté, dans la mesure où un africaniste français qui inscrit sa citoyenneté en France n'a pas la même responsabilité ou "accountability» face à ses travaux qu'un chercheur africain qui inscrit sa citoyenneté en Afrique. L'utilisation positive ou négative des recherches de ce dernier dans le débat public local est d'une intense réalité ; partant: une contrainte supplémentaire, que ne connaissent pas les chercheurs du Nord qui rentrent dans leur pays après une mission. Pour autant, Olivier de Sardan ne défend aucune «spécificité épistémologique » africaine. Les règles du jeu des sciences sociales sont les mêmes partout. Dans la mesure cependant où elles sont abordées au Nord ou au Sud avec de profondes inégalités de ressources, d'environnement et de compétences, il importe de les clarifier rigoureusement (OLIVIER DE SARDAN 2008).

13. Pour nuancer ces simplifications, voir HARRISON (2003) et l'article de Jean-Louis TRIAUd (dans ce numéro).

14. Voir la revue Islam et Sociétés au sud du Sahara fondée par Triaud en 1987. 
Depuis une vingtaine d'années cependant, il s'est produit un changement signifiant (et salutaire au vu des problèmes signalés précédemment) avec l'émergence de ce que Louis Brenner (1999) a appelé « une voix musulmane africaine à l'université »- dont Amadou Hampâté Bâ, partenaire de recherche de Théodore Monot puis auteur en son nom propre de divers travaux dont Vie et enseignement de Tierno Bokar (Bâ 2004) fut peut-être un précurseur atypique et isolé ${ }^{15}$. Cette voix musulmane est d'abord le fait de dignitaires religieux qui réagissent et parfois objectent publiquement aux écrits des chercheurs occidentaux sur leurs communautés. Pour Marcus et Fischer, repris dans l'introduction de l'ouvrage When They Read What We Write: The Politics of Ethnography, «ce lectorat "indigène" [native] et potentiellement critique » ne constitue rien moins que «l'incitation la plus puissante à l'élan expérimental contemporain de l'écriture anthropologique, à la fois comme ethnographie et critique culturelle » (Marcus \& Fisher 1986, cités dans Brettell 1993: 2). Ainsi Brettell (1993: 3) qualifie-t-elle son émergence de « révolution du lectorat» (« revolution in readership»).

Mais la voix qu'évoque Brenner est aussi et surtout le fait de chercheurs musulmans africains qui investissent le domaine des études sur l'islam en Afrique en alliant exigence critique et respect des sensibilités religieuses ${ }^{16}$. $\mathrm{Si}$ ces chercheurs musulmans ont initié de nouveaux canaux d'échange en direction de leurs confrères occidentaux non musulmans ${ }^{17}$, ces derniers - désormais formés dans le bain des controverses sur l'orientalisme et le regard euraméricain sur l' «Autre » engendrées par l'œuvre d'Edward Saïd et ses critiques (Freitag 1997) — ces derniers donc, au rang desquels je me situerais, ont emboîté le pas et tendent aujourd'hui, en retour, à montrer plus d'empathie envers l'islam et les sociétés musulmanes. Contre la tendance qui ferait que «plus que tout autre phénomène social, l'étude de la religion tend à refléter les présupposés du chercheur ${ }^{18}$, les spécialistes sur l'islam en Afrique, tous horizons confondus, relancent et tentent de dépasser le vieux débat - en rien spécifique aux aires culturelles (McCutcheon 1999 ; Arweck \& Stringer 2002) — quant à savoir lequel des deux points de vue ou positionnalités «émique »/insider d'un côté et «étique »/outsider de l'autre constitue qui un atout, qui une entrave pour la connaissance scientifique du groupe religieux étudié ${ }^{19}$.

15. Voir aussi le personnage moins connu mais non moins prolifique que fut Shaykh Muusa Kamara (1964-1945) : BOUSBINA (1992) et KAMARA (1998).

16. Launay (2006) note toutefois avec une pointe de regret que ces nouveaux chercheurs africains sont rarement anthropologues et plutôt historiens.

17. Sans être dénuées de pertinence, ces catégories ne sont pas entièrement satisfaisantes : trop réifiantes, elles mériteraient d'être nuancées.

18. Olivier de SARdan (1988 : 537) citant Mary Douglas ; repris dans van DiJK \& PelS (1996: 247).

19. Voir Olivier De SARDAN (1998) sur l'usage de ces catégories reprises de Pike et de Harris, plus en vogue dans le monde anglo-saxon qu'en France. Voir Clough (2006) pour une analyse réflexive d'un universitaire américain sur le rôle qu'a joué son bagage catholique dans sa compréhension de sociétés musulmanes au Nord Nigeria. 
Nourrie par ces nouveaux questionnements qui en appellent moins à des réponses claires qu'à un cheminement prudent, j'en suis venue à faire miennes les recommandations de Brenner (1999) pour qui «[1]e vrai défi qui se pose aujourd'hui à nous, chercheurs [non-musulmans], est de cultiver ces germes de sensibilité et d'empathie [envers les musulmans] sans lesquelles il ne peut y avoir de développement fructueux de notre domaine, en dépit de tout l'argent investi dans les nouveaux instituts » pour l'étude de l'islam en Europe et aux États-Unis.

Le laboratoire d'Abidjan : éveil à l'empathie et tentation polyphonique

Mon travail doctoral sur la communauté musulmane d'Abidjan a représenté pour moi une sorte de laboratoire face au défi de gestion de l'empathie et donc un précédent à la relation avec Vissoh. Car entre éprouver une gratitude débordante face à l'accueil généreux qui m'était réservé et négocier la bonne distance pour une intersubjectivité empathique mesurée, il y avait un écart qu'il n'était pas évident à combler (Olivier de Sardan 2008) ${ }^{20}$.

J'avais délibérément choisi de porter mon attention sur une communauté religieuse peu étudiée, donc sur un terrain peu défriché, une situation qu'expliquait en partie le fait que cette communauté, longtemps minoritaire, avait évolué à l'écart du pouvoir d'État dans un contexte sociopolitique national qui, jusqu'au milieu des années 1990, était encore plutôt paisible. Mon ambition visait alors à reconstituer du dedans l'évolution de cette société islamique discrète, oubliée des grands narratifs de l'histoire nationale. Pour ce faire, et même si j'ai aussi consulté des archives, l'apport des sources orales et des enquêtes participantes a été déterminant. Mon défi fut donc de produire un travail rigoureux ne dévoyant pas des exigences universitaires (pas question de laisser l'empathie devenir débordement fusionnel et abandon de l'esprit critique), mais en lequel pourrait aussi se retrouver une majorité de mes interlocuteurs qui avaient tacitement accepté que je devienne dépositaire de leur mémoire collective (un rendu de type surplombant, théoriciste, dans une distance froide, se serait peut-être imparfaitement acquitté de cette dette que j'interprétais instinctivement comme une forme de responsabilité morale).

Ce défi fut singulièrement compliqué par l'embrasement des troubles politiques en Côte-d'Ivoire après 1999 et les violences physiques et morales qu'eurent à subir les musulmans du pays, amalgamés au groupe des étrangers (Miran 2006). Au vu des insanités anti-islamiques et xénophobes qui se publiaient dans la presse ultranationaliste, encore aggravées après les événements du 11 septembre 2001 et le coup d'État manqué du 19 septembre

20. L'absence d'affinité de l'anthropologue pour le milieu étudié n'est pas moins problématique (FANCELLO 2008). 
2002 qui provoqua une quasi-guerre civile (et la désertion de bien des chercheurs occidentaux du terrain ivoirien), je me sentis investie d'un devoir urgent à témoigner sereinement, en élargissant mon public imaginaire à toute la société ivoirienne. La tâche était malaisée. Prise dans l'étau de passions contraires, je ne voulais ni me faire le relais du sens de mission qu'animaient les milieux musulmans que j'avais côtoyés et dont j'étais restée proche, ni être prise à parti par quelque considération politico-idéologique que ce soit. Un risque était de me voir interdire l'accès au terrain. L'empathie en sa face périlleuse...

C'est ainsi qu'insensiblement, je mis à l'essai quelques stratégies concrètes (qui ne portent pas toutes le blason de l'originalité !) pour essayer de traduire mon empathie de manière équilibrée à l'écrit et en actes. Sans tomber dans le tout empirisme, j'eus le souci que mes textes rendent le contexte au plus près. Il m'importait en premier lieu que mes travaux se fassent l'écho d'une pluralité de voix musulmanes, souvent en citation directe, pour donner du coffre à la dimension polyphonique de la communauté elle-même, jusqu'au sein de courants « homogènes »; et ce, pour moduler également la trame monologique de ma construction narrative et glisser un bémol aux vertus nécessaires de la modélisation. Pour écrire une histoire à visage humain par-delà les processus macro- et supra-locaux, j'ai multiplié les profils biographiques, sans chercher à gommer ce qui pouvait paraître relever de contradictions. Et pour donner chair au terrain, en l'occurrence à l'environnement urbain d'Abidjan, y compris matériel, je n'ai pas été avare de détails, au risque de faire ployer le lecteur sous la prolixité du texte. Ma banque de données fut les notes de terrain prolifiques que j'avais prises et saisies au quotidien à l'imitation d'un journal ethnographique, en adjoignant à la description des récoltes d'information des appréciations délibérément personnelles et subjectives sur les émotions et les impressions qu'imprimaient en moi les personnes rencontrées et les situations vécues, ce qui m'aida à lever le voile sur l'alternance entre immersion et distanciation (Kleinman \& Copp 1993 ; Olivier de Sardan 2008). Je m'imposais finalement de référencer de manière aussi précise et exhaustive que possible mes sources et notamment les propos de mes interlocuteurs, à la fois pour leur témoigner publiquement de la reconnaissance et pour que sur ce sujet qui restait peu couvert, ma thèse et sa publication révisée puissent aussi servir de base de données «traçables » (contre l'idée qu'il faut croire l'anthropologue sur parole «parce qu'il y était»). Ceci, pensais-je, permettrait à d'autres de situer aisément les chaînons manquant et les failles d'interprétation et d'aller de l'avant dans les recherches. Sauf rares exceptions tombant sous le coup du bon sens et forte de l'encouragement de quelques guides religieux, je n'eus donc pas recours aux pseudonymes. Recul à l'appui : les acteurs de la communauté musulmane d'Abidjan n'ont exprimé que de la satisfaction à se voir cités en nom propre dans mon livre, à l'instar des informateurs calédoniens d'Alban Bensa (2007). 
Il se trouve que la communauté urbaine que j'avais choisi d'étudier jouit d'une frange importante d'intellectuels musulmans francophones. Nombre de leurs associations et comités de gestion de mosquées s'avérèrent relativement bien organisés et souvent dotés d'ordinateurs. La donne facilita mon choix de diffuser largement les premières moutures de mes écrits, sous forme papier ou en version numérique, via transferts par clé usB et attachements par email, sur place ou à distance. Je sollicitais des réactions qui me permirent de faire des ajouts et quelques révisions, sans effleurer l'écueil de la censure. L'exercice de redoublement d'écoute s'avéra salutaire contre un penchant à la surinterprétation de type rhétorique, dont je pris conscience de la (dé)mesure des capacités déformantes...

Le Ph.D. obtenu, j'offris le volume en m'adressant symboliquement au Conseil supérieur des imams de Côte-d'Ivoire (COSIM), qui, par goût du cérémoniel, orchestra la remise dans la mosquée qui servait de siège à l'institution, en présence des grands imams de la ville réunis en conclave hebdomadaire. Dans la même veine, quand le livre fut publié quelques années plus tard, l'Association des élèves et étudiants musulmans de Côted'Ivoire (AEEMCI) organisa une table ronde à laquelle furent aussi conviées, à ma surprise, la presse et la télévision nationales : j'eus à m'expliquer ardemment sur le projet et son résultat. La scène avait des effluves de soutenance et j'eus d'ailleurs droit à un diplôme d'honneur, que je pris comme un accusé de réception du «rendu provoqué » de mon travail.

Pour éviter le partisanisme, je fis ensuite la démarche d'aller présenter mon livre à des journalistes de la mouvance ultranationaliste et j'ai enregistré des émissions à la fois à la radio islamique et à la radio catholique. Autre manière de «rendre »: au fil des ans, mes interlocuteurs abidjanais ont dirigé vers moi une poignée d'étudiants ivoiriens de l'Université de Cocody, issus de familles musulmanes, qui avaient formé des projets de recherche sur l'islam en Côte-d'Ivoire. Le dialogue avec ces maîtrisards et thésards se poursuit. Ma priorité est aussi d'encourager les (rares) acteurs musulmans qui m'ont fait part de leurs désirs personnels d'écriture. Dans les deux cas, l'invite à publier porte quelques fruits.

La rencontre avec El Hadj Charif Vissoh : (auto)biographie d'une collaboration

La rencontre avec El Hadj Akan Charif Vissoh, imam béninois d'Allada, m'a permis d'aller plus loin dans le désir de provoquer l'échange et de matérialiser le croisement des regards entre le chercheur et l'acteur religieux. L'idée d'écrire un article à deux n'a pourtant germé que lentement, dans un chassé-croisé laborieux, avant de s'imposer dans la douceur de l'évidence.

Le doctorat mené à terme, je développais un nouveau projet de recherche sur les convertis à l'islam dans la région du golfe de Guinée et à la faveur 
d'un séjour à Cotonou en 2003, je me fis présenter le président-fondateur de la Ligue islamique béninoise pour la promotion et le soutien des convertis musulmans (LIBPSCOM), en la personne d'El Hadj Vissoh. Nous eûmes un long entretien au cours duquel on parla beaucoup des convertis à l'islam dans le sud-Bénin et du travail de la LIBPSCOM mais pas beaucoup de Charif Vissoh lui-même - sans doute, de ma part, par besoin de lumière sur un contexte qui m'était étranger et plus encore par crainte pudique de messéance, en cette première rencontre, face au territoire de l'intime. Au moment où l'entretien prenait fin et où je balbutiais les remerciements d'usage, Vissoh sollicita l'initiative de la parole. Il souhaitait me raconter l'histoire de sa vie et de sa conversion, convaincu que le détour m'aiderait à comprendre les difficultés que traversent les convertis au sud-Bénin, difficultés liées principalement aux tensions avec les familles d'origine mais aussi à la relative indifférence voire au mépris dans lequel les musulmans nés dans l'islam tiendraient toujours leurs coreligionnaires néophytes.

D'emblée donc, il y eut entre nous alternance de tours de parole et de temps d'écoute, de rôles plus actifs et plus passifs ou réactifs, dans un même élan de curiosité avide et consciencieuse, une même visée pédagogique pardelà le nouement relationnel. Dès le début aussi, les propos de Vissoh alternaient un double niveau de langage puisqu'il parlait certes de son expérience personnelle et même fort intime, mais dans l'objectif in fine de porter témoignage et d'interpeller les musulmans nés dans l'islam au nom collectif des convertis. Je compris par la suite qu'il avait du mal à se faire entendre à la fois de sa propre famille qui n'avait pas approuvé sa conversion et des principaux dirigeants musulmans de la place, et qu'il avait besoin d'un intermédiaire dans la poursuite de ce qu'il voyait comme son devoir de mémoire et sa mission de défense et de promotion des convertis au nom de sa conception réformiste de l'islam. Je compris également qu'il révérait la perspective de pouvoir coucher son histoire et celle d'autres convertis sur papier, mais qu'en homme d'action et en pédagogue de l'oralité, tout lettré qu'il était par ailleurs, Vissoh avait besoin d'être accompagné dans ce passage à l'écriture. Nos complémentarités furent un atout solide.

Il s'est suivi de notre rencontre un monologue fleuve de plus de quinze heures sur une semaine, dans lequel je suis minimalement intervenue, toute à la prise de notes que Charif, en bon enseignant coranique, supervisait de près, en m'épelant certains noms, en me demandant de temps à autre si je suivais bien et en marquant de longues pauses d'émotions contenues à l'évocation de moments difficiles, notamment la mort de sa première épouse $^{21}$. Les silences, assurément, nous rapprochèrent plus encore que les

21. Mon choix initial fut de m'abstenir d'enregistrer parce que l'expérience m'a enseigné que face à un interlocuteur inconnu, la parole de l'informateur tend à être plus libre et plus vive sans enregistreur, non pas tant (ou pas seulement) par autocensure que par respect pour la parole gravée amenée à durer, dont on ne sait ce qu'elle peut devenir. 
mots. Je saisis alors avec une limpidité déconcertante l'écart qu'il pouvait y avoir entre les intérêts de recherche de l'observatrice extérieure que j'étais - et qui se croyait pourtant pas trop mal préparée - et les préoccupations majeures, je dirais presque existentielles, de l'acteur lui-même, objet de ma recherche et de mon essai de maïeutique, que je n'avais pas su questionner.

Je compris tout de suite que le matériau brut du témoignage d'El Hadj Vissoh, pas toujours bien agencé chronologiquement mais très riche en détails, éclectique par moments mais supporté par une vraie trame narrative et une vraie motivation sociale et religieuse, était un document de valeur qui avait échoué entre mes mains sans que je l'aie vraiment sollicité. Je compris tout de suite encore que je m'étais implicitement engagée, en écoutant ce témoignage, à le travailler et à le publier : donnant-donnant. En revanche, cela me prit plus de deux ans pour faire sens de ce que je devais en faire, pour comprendre comment exprimer au mieux nos empathies réciproques et les intérêts différents mais synergiques qu'avaient révélés nos échanges.

De retour aux États-Unis où j'enseignais alors, une première étape a été de saisir fidèlement les notes de nos entretiens sur ordinateur. Le email, bientôt relayé par de longues conversations téléphoniques transatlantiques, fut mis à contribution pour l'envoi du texte à Vissoh avec quelques questions de précision, pour commentaire ${ }^{22}$. Vissoh produisit en retour un texte de sa main, assorti de commentaires oraux, avec des corrections de détail mais aussi des rajouts, une meilleure contextualisation de certains épisodes, une actualisation de son histoire (car sa vie restait riche en événements depuis notre rencontre : ainsi sa nomination imamale et la mort de son père) et des instructions générales sur ce qu'il importait de bien mettre en valeur.

Une deuxième étape a été l'écriture d'une communication que je donnais seule, en anglais, à une conférence au Zentrum Moderner Orient de Berlin sur le thème «Conversion, modernité et individualité » (2005). Ma présentation parlait essentiellement de Charif Vissoh mais dans un style bien sûr très différent du témoignage retouché qui en était la source. C'était surtout une analyse synthétique, qui intégrait en outre des données que j'avais recueillies auprès d'autres convertis à l'islam en Côte-d'Ivoire et au Ghana, pour parler aussi de changement social et de dimension collective par-delà la seule expérience d'un individu. Je fis bien sûr suivre le papier, qui moissonna de nouveaux commentaires de la plume de Vissoh. Mais ce dernier réagit surtout en se piquant à son tour au jeu de la recherche. Il s'improvisa enquêteur auprès de certains de ses proches pour faire la lumière sur des

22. Depuis que ma mobilité a été sérieusement entamée par la naissance de mes enfants (une contrainte non politique mais non négligeable d'accès au terrain !), je pratique en plus des traditionnels séjours de terrain, des entretiens d'enquête par téléphone. Indéniablement, ce projet collaboratif n'aurait pu être mené à bien sans l'appui des nouvelles technologies de l'information. 
questions empiriques restées dans le flou et il me fit part des données récoltées : ainsi la date et les circonstances de la conversion au catholicisme de son père. Il poussa l'initiative jusqu'à faire des recherches d'archives et trouva un manuscrit ancien auprès d'un des membres de son association, descendant d'une grande famille de marabouts jadis attachée au service du roi du Danxomé : le manuscrit apportait quelques éclairages historiques sur les relations entre les musulmans minoritaires et le pouvoir « infidèle » dans la région d'origine de Charif. C'est au fil de ces échanges que je compris que le nom d'El Hadj Vissoh devait figurer dans la signature du texte en cours d'écriture, eu égard à son implication active et soutenue. La reconnaissance de dette était aussi désir d'élargir l'horizon de l'échange, en troquant le souci d'un «droit de réponse » contre un appel à co-contribution. Vissoh s'étonna de ma proposition mais l'accepta avec enthousiasme, en s'engageant à assumer sa part de négociation dans notre travail à ré-imaginer pas à pas.

Je pensai d'abord faire marche arrière et proposer à Charif de publier son texte en tant que source primaire, dans la lignée des « autobiographies à deux voix ${ }^{23}$ qui enrichissent régulièrement la littérature en histoire (orale) et en anthropologie de l'Afrique - et bien au-delà, comme le montre l'exemple des études latino-américaines où le genre dit «testimonio », ou récit de témoignage d'un acteur subalterne à la première personne, connaît un regain de succès (Gugelberger 1996). Je pensai aux modèles que pouvaient représenter, pêle-mêle, Baba of Karo, A Woman of the Muslim Hausa de Mary Smith (1954), Nisa, The Life and Words of a Kung Woman de Marjorie Shostak (2000), Le dernier éléphant, Histoire d'un chasseur kenyan de JeanLuc Ville et Abajila Guyo (2004) ou encore les trois volumes publiés par Bernard Salvaing : les deux tomes d'Almamy co-écrits avec Almamy Maliki Yattara (2000, 2003a) et Une vie au Fouta Djalon (2008) co-écrit avec alhadji Thierno Mouhammadou Baldé. Ce genre autobiographique honorait l'exigence principielle de respect dû aux acteurs que le sociologue Harold Garfinkel, figure de proue du courant ethnométhodologique et de l'école de Chicago, avait été l'un des premiers à systématiser (Garfinkel 1967) ; les études sur les femmes et le genre aux États-Unis amplifièrent ce souci (Wolf 1992). Depuis lors, cette approche s'était consolidée dans le courant de l'anthropologie interprétative «du point de vue des autres » (ou «des indigènes » : Geertz 1973, 1983 ; Blundo \& Olivier de Sardan 2003) et dans celle de l' «éthique narrative» issue des controverses sur l'orientalisme.

Je me suis ensuite ravisée en réalisant qu'un récit linéaire risquait d'oblitérer les niveaux de lecture du témoignage de Vissoh qui dépassait la seule narration événementielle et sa mise en contexte social : à savoir le cadre de la production du texte et la représentation que Vissoh s'en faisait et se faisait de lui-même. Pour l'imam d'Allada en effet, s'investir dans cet écrit relevait d'un acte de foi, à la fois étape de sa conversion et tract réformiste.

23. Je reprends l'expression du titre d'un article de SALVAing (2003b). 
Mais pour nuancer et complexifier le tout, Charif n'était pas sans humour et recul sur lui-même : ainsi s'autocritiquait-il parfois obligeamment sur ses propres postures, en faisant se croiser ses regards pluriels de sujet individuel, de membre du groupe ethnique mahi, de simple croyant musulman, d'imam et de président d'une association de convertis, chaque regard ayant son registre propre, avec sa cohérence et ses limitations, dont il était bien conscient.

De son côté, Vissoh tenait à conserver les interludes comparatifs sur les convertis ivoiriens et ghanéens que j'avais entretissés à sa narration biographique dans la version de Berlin, parce que cela servait son propos de parler au nom des convertis. Que je puisse témoigner à l'appui de mes enquêtes hors du Bénin d'expériences de convertis similaires à celles de Vissoh, consolidait peut-être la légitimité des propos de ce dernier, à qui reproche avait déjà été fait de donner trop de coffre à ses difficultés et d'aller vite en besogne à les généraliser. Sans surprise, on tomba ensuite d'accord pour élargir la comparaison aux conversions minoritaires d'Européens à l'islam. Sur un autre plan, Vissoh était parfaitement au fait des enjeux d'une parole publique puisqu'il exerçait, outre les fonctions d'imam et d'enseignant coranique, celle de journaliste à la seule radio islamique de Cotonou, «La Voix de l'Islam » de la mosquée zongo. Sans fausse note, on s'accorda finalement pour co-écrire et co-signer l'article, conscients que l'aventure reposait avant tout sur notre amitié et le pari de dépasser, ensemble, nos limitations réciproques.

Ce choix posait en définitive le pari de dépasser — ou brouiller plus avant - la dialectique classique entre interpréter et décrire (Bazin 1996) ${ }^{24}$ : il nous transportait, au-delà du dialogue, vers la collaboration ethnographique. Si le retour en grâce de l'ethnographie collaborative est un phénomène récent, le courant a des racines déjà anciennes, comme évoqué en introduction. Nulle prétention à l'innovation : l'aventure a des précédents (mais le philosophe André Comte-Sponville [2003] ne dit-il pas avec humour : «Ce n'est pas l'originalité que je cherche : une idée que personne n'aurait jamais eue, cela a toute chance d'être une sottise!»). L'œuvre déjà citée de Luke Lassiter et notamment son Chicago Guide to Collaborative Ethnography (2005b) documente l'histoire des pratiques de collaboration et plus spécifiquement de co-écriture aux débuts de la discipline, lesquelles furent d'une richesse particulière dans le domaine des études sur les Amérindiens d'Amérique du Nord. Les collaborations entre Franz Boas et son «partenaire privilégié ${ }^{25}$ tlingit/kwakiutl George Hunt (Boas \& Hunt 1895), entre Alice Cunningham Fletcher et son partenaire omaha Francis La Flesche (Fletcher \& La Flesche 1911) et entre Paul Radin et son partenaire winnebago Sam Blowsnake (Blowsnake \& Radin 1920) en sont d'illustres exemples.

24. Voir aussi les archives sonores de la journée d'études « Jean Bazin (1941-2001) : Terrains africains. Une autre anthropologie? », sur <http://lodel.ehess.fr/ceaf/ document.php ?id=709>.

25. Selon l'expression d'Olivier DE SARDAN (2008). 
Sur l'Afrique, l'important ouvrage de Marcel Griaule Dieu d'eau, entretiens avec Ogotommêli (1948) relève sans doute de cette catégorie, quelles qu'aient été les critiques pertinentes qui furent ensuite soulevées sur le rapport de l'anthropologue français au terrain dogon (Clifford 1983b; Vanbeek 1991). Bien que non estampillée d'une double signature, l'œuvre maîtresse de Bernard Maupoil, La géomancie à l'ancienne Côte des Esclaves (1943), repose également sur une étroite collaboration avec l'interlocuteur principal que fut l'un des fils Nondichao de la grande famille de devins musulmans auprès des rois d'Abomey. De même, la monographie Prophétisme et thérapeutique (Piault 1975) doit beaucoup aux affinités électives entre Jean Rouch et Albert Atcho de Bregbo. Il y eut aussi les diverses collaborations entre Théodore Monod et Amadou Hampâté Bâ (Triaud 2009), et la liste est loin d'être exhaustive... Plus proche de nous, cette tradition collaborative a continué de produire quelques ouvrages, dont celui de Luke Lassiter et al., The Other Side of Middletown : Exploring Muncie's African American Community (2004), et celui de Field et al., Abalone Tales : Collaborative Explorations of Sovereignty and Identity in Native California (2008a), tous deux cosignés avec une pluralité d'acteurs locaux issus des communautés étudiées. Bien que je ne prisse la pleine mesure de cette filiation que rétroactivement, c'est dans cette tradition que je proposais intuitivement à Charif Vissoh de nous inscrire, modestement.

Notre article prit alors lentement forme, au fil de séries de révisions entre le Bénin et les États-Unis. Une première décision fut de revenir au français, langue du public musulman béninois auquel Vissoh, par ailleurs anglophone, voulait s'adresser ; cela minimisait aussi les risques de méprise linguistique entre nous. Le français oral de Vissoh fut lissé pour être de bonne tenue à l'écrit et donc refléter le niveau d'éducation avancé de mon co-auteur. On négocia la structure du texte. Il m'incomba de produire le premier jet. Vissoh le corrigea partie par partie, grâce aux outils informatiques et à l'incontournable appui du téléphone. Il supervisa ensuite l'intégration des révisons. Par tempérament ou par décision, nos postures étaient conciliantes l'un vis-à-vis de l'autre et nous n'eûmes pas de vrai différend. Néanmoins, on débattit longuement de la pertinence d'évoquer les événements les plus intimes de la vie de famille de Vissoh (il y tint, je m'alignais) et de l'opportunité d'inclure la marée montante des nouveaux détails qu'accompagnait chaque relecture (Charif acquiesça quelques coupes, l'article final n'en fit pas moins 49 pages). On discuta aussi du principe d'évoquer entre les lignes plutôt que frontalement certaines des récriminations de Vissoh à l'encontre de ses coreligionnaires, pour ne pas prêter le flanc à la polémique. Bien qu'habitué au franc-parler, Vissoh concevait sa prise de parole comme une invitation au dialogue et au changement et non comme une fermeture: on s'entendit également sur ce point.

Une des dernières étapes de notre collaboration porta sur le choix des photographies devant illustrer le texte. Charif trouvait banale la photo que j'avais prise de lui à l'improviste à Cotonou devant les bureaux de la radio 
«La Voix de l'Islam» (Miran 2009 : 681). Il me fit alors la surprise de se faire tirer de superbes portraits de plain-pied en studio, à ses frais. Éclatants de couleurs, certains portraits le représentent avec tout le regalia de ses fonctions d'imam - abaya de la Mecque, turban ou keffieh (pp. 659, 691). Un autre le montre en tenue typique des musulmans du nord du Bénin boubou en bazin et calotte assortie (p. 683). La photographie la plus impressionnante le figure en prière, assis sur un tapis, chapelet à la main (p. 659). Vissoh reconnut volontiers s'être mis en scène et choyer son image publique. Mais il débouta toutes mes surinterprétations : le décor des couleurs n'était pas de son cru mais du photographe et s'il avait choisi ces tenues et ces poses, c'était aussi pour moquer les clichés populaires qui engoncent les imams d'une part et les musulmans de l'autre dans des «look-s » figés et en partie « ethniques ». Sans disconvenir radicalement de l'importance visuelle des choses extérieures - il se prêtait d'ailleurs au jeu - Vissoh entendait aussi faire passer un message plus nuancé, à savoir qu'il n'est pas requis de porter le boubou des Sahéliens pour être musulman, ni de se conformer au protocole « officiel » de l'imam saoudien ou hausa pour diriger la prière en islam. Et pour étayer son propos, Charif ressortit simultanément de vieilles photos de ses albums personnels, où on le voit jeune homme, en chemise et en pantalon à l'occidentale, sur l'une faire prier sa femme et leurs trois enfants à domicile (p. 670), sur l'autre prendre part à une conférence islamique (p. 667). Si tant est qu'il y a des affinités complexes entre l'extérieur/le visible et l'intérieur/le caché, l'ensemble de ces photos permet d'imaginer les ruptures et les continuités qui ont jalonné le cheminement religieux de Charif Vissoh, de sa conversion en 1974, à la publication de son récit de conversion trente-cinq ans plus tard. Nos discussions sur la polysémie des photographies faisant se croiser la représentation de soi, privée ou publique, et les perceptions des autres, populaires ou savantes, sont à l'image de notre co-façonnage du texte, pétri d'efforts d'intercompréhension.

$*$

Forces et faiblesses de notre aventure collaborative

Si notre expérience de co-écriture fut gratifiante de nos deux côtés, force est de reconnaître qu'aux plans méthodologique et épistémologique, elle soulève autant de questions qu'elle n'avance de solutions. S'impose donc un bilan critique de ses atouts et de ses limites.

Qu'en est-il d'abord des limites ? L'exercice ambitionne de faire dialoguer deux voix hétérogènes et parie sur le fait que l'écho qu'elles se renvoient puisse produire un entrelacement discursif si ce n'est nouveau du moins quelque peu différent, décalé, éclairant. Mais le risque est toujours présent qu'une voix finisse par absorber l'autre. Cela pourrait être le résultat 
d'une manipulation de mauvaise foi, si d'aventure Vissoh s'était servi de moi, par exemple, pour régler des comptes et faire la politique de sa promotion personnelle ou si tout ne fût qu'une mise en scène pour tenter de me convertir. Mais le risque que la voix de l'ethnologue, surtout s'il est occidental, coopte celle de l'acteur, surtout s'il est africain, est plus grand encore, quoique plus subtil. Ainsi me suis-je demandé si je n'imposais pas en fin de compte sur la voix de l'imam d'Allada ma propre représentation culturelle de sa personne, de sa conversion, de sa société et de sa religion, et ce de manière hégémonique, c'est-à-dire en allant jusqu'à soutirer l'approbation d'El Hadj Vissoh... Il se peut aussi que je fusse victime, en toute bonne foi, d'une sorte de ruse d'authenticité : je n'aurais fait que me cacher derrière une façade de débat qui n'en serait pas un, en prétendant accéder au plus près de la «vérité de l'indigène " ${ }^{26}$ (McBeth 1993).

A minima, il faut reconnaître que les relations de pouvoir en termes d'inégalités dans l'économie de production de notre article n'ont pu être effacées entre nous. Vissoh n'a pas été directement rétribué pour son travail et il était établi d'avance qu'il n'y avait pas d'enjeu de copyright autour de la publication : notre collaboration n'a reposé que sur des motivations extra-matérielles. Vissoh n'a pas été mon assistant. De ce côté, au moins sur un plan symbolique, nos relations furent sur pied d'égalité. Mieux, en termes d'autorité intellectuelle, fort de sa bonne formation arabo-islamique, de son expérience d'enseignant et de son autorité de guide religieux, Vissoh avait la main haute sur certains points discutés. Il n'empêche que les moyens financiers dont je disposais pour concrétiser notre projet étaient, bien que modestes, sans commune mesure avec ceux de mon collaborateur; je ne pus que très partiellement et très imparfaitement combler ce fossé entre nous. Le constat peut être élargi à l'ensemble du public auquel Vissoh voulait s'adresser: la publication finalisée est trop coûteuse (quand elle est disponible) pour trouver acheteur au Bénin. Le support numérique et les photocopies pallient faiblement cet état de fait.

Il est possible — si l'idée ne relève pas d'un prêt-à-penser paresseux que ces déséquilibres dans la politique et l'économie de production de notre travail aient été vaguement amortis par l'altérité de genre entre nous, l'altérité homme-femme. Pour le dire inversement: une collaboration entre un homme «du Nord» et une femme «du Sud» porterait peut-être plus de risques de domination d'une voix sur l'autre ${ }^{27}$. La (bonne) volonté de situer l'autorité narrative dans le processus dialogique n'a pas valeur de résultat ${ }^{28}$.

26. Comme me le fait remarquer Robert Launay, on peut se demander plus largement si certains courants anthropologiques, notamment nord-américains, ne produiraient pas une fétichisation de la « voix », reposant sur l'idée (faussement ?) naïve que le fait de citer directement ses collaborateurs est simultanément source d'authenticité et de pouvoir.

27. Pour une opinion discordante, voir BERLINER (2008).

28. Selon la remarque pertinente d'Olivier de Sardan, en co-signant un article avec Vissoh, je le fais jouer volens nolens au jeu des sciences sociales, le mien (même 
Postulons la réussite de notre pari de débattre de manière pas trop hégémonique: la critique se déplace alors à un niveau presque littéraire, au niveau de l'écriture même et de sa réceptivité auprès du lecteur. Étant donné que notre texte mêle nos deux voix sans les différencier systématiquement explicitement, mais qu'on ne parle pas toujours d'une même voix, qu'il y a des tours de parole, différents niveaux de lecture, qu'on a des objectifs et des audiences différents en vue et que le texte a même un côté performatif : la question reste ouverte de savoir si cela ne crée pas un texte trop alambiqué, illisible, inexploitable... N'aurait-on pas produit, malgré nous, un cocktail indigeste de diverses tendances en anthropologie postmoderne, à la fois réflexive, critique, interprétative, littéraire, etc. ? La polyphonie peut devenir cacophonie ! Le verdict reviendra aux lecteurs, tant dans les milieux universitaires que dans le milieu musulman béninois.

Autre limite : il va de soi que cette expérience n'est pas toujours possible. Ancrée dans la tradition certes discrète et périphérique mais ancienne de l'ethnographie collaborative : l'histoire atteste qu'elle est reproductible par d'autres, qu'elle n'est pas irréductible aux conditions particulières de notre échange, si tant est qu'il y a, minimalement, réciprocité dans l'empathie, c'est-à-dire respect absolu de part et d'autre de l'altérité de chaque interlocuteur. L'illusion de relation fusionnelle ou de métissage enchanté des cultures eut été un obstacle (Mary 2000). Si j'eusse tenté la conversion à l'islam pour mieux comprendre le cheminement de Vissoh, c'eût été une autre aventure $!^{29}$. A contrario, pour mieux écouter et accepter l'autre, il nous fallait intégrer nos limites à la compréhension d'autrui, notamment pour moi la part d'indicible dans les motivations de la conversion religieuse de Vissoh. Le fait demeure que même quand ces conditions sont remplies, la co-écriture n'est pas promise à devenir la norme. Elle ne peut prétendre être une révolution épistémologique. Sans compter qu'on ne peut pas dialoguer avec les morts ${ }^{30}$, je doute qu' on puisse mener à bien un travail de coécriture avec un mafioso! André Mary (2000) souligne qu'un tel travail serait de même difficile à envisager avec un pasteur évangélique dont le

s'il a été d'accord pour y participer). S'il m'avait fait intervenir dans une réunion d'une association islamique, il m'aurait fait jouer à un autre jeu, le sien. Pas de hiérarchie de valeur ou de légitimité entre les deux : ce sont simplement des registres différents. Cette réalité est clairement une limite fondatrice de l'anthropologie collaborative.

29. Sur la conversion de Benetta Jules-Rosette aux Apôtres de John Maranke, voir JulES-RosetTe (1976). Voir également MARY (2000 : 131). Sur l'expérience initiatique de Pierre Fatoumbi Verger au Brésil, voir Souty (2007). Pour une réflexion critique sur le choix méthodologique d'un anthropologue américain non musulman de participer à la prière musulmane sur son terrain indonésien (et donc de passer pour un musulman aux yeux de ses hôtes), voir LuKENS-BuLL (2007).

30. Les historiens et notamment ceux qui étudient le contexte colonial partagent le souci de valoriser les sources «indigènes » et d'exhumer la voix des «indigènes » dans les sources exogènes, surtout quand les premières sont rares, mais stricto sensu, leur travail exclut la possibilité d'une co-écriture. 
régime de vérité délégitime foncièrement celui du chercheur non converti (Fancello 2008). Mais la co-écriture serait tout aussi difficile à imaginer avec un paysan subsaharien, non intellectuel, analphabète, voire non europhone, surtout si la langue de publication n'est pas vernaculaire. Cela réduit considérablement, hélas, le champ des possibles de ce genre d'échange, qui ne peut orchestrer de face-à-face que quand l'altérité des interlocuteurs n'est, paradoxalement, pas incommensurable. Comme le reformulait Olivier de Sardan: les recherches qui se prêtent le mieux au pari de la co-écriture relèvent d'un «type » assez spécifique portant sur des représentations érudites ou des savoirs spécialisés, parce que ces derniers relèvent d'emblée de la coproduction de savoirs par deux modes d'érudition distincts mais ayant quelques affinités. Les recherches en socio-anthropologie des savoirs populaires, des stratégies politiques ou des institutions s'accommoderaient mal de la co-écriture. Au sein même de ce «type» de recherche, la coécriture n'est qu'une option possible, sans monopole de légitimité.

Pour autant, la démarche n'est pas sans atouts, même si ceux-ci restent des paris plus que des acquis. La poursuite du travail de terrain dans l'écriture, le renvoi du travail d'écriture vers l'interlocuteur sur le terrain, le rapprochement de l'insider et de l'outsider, du musulman et du non-musulman, de l'ethnologue et de l'imam: tous ces croisements peuvent nourrir une approche potentiellement féconde et inventive à même d'élargir notre compréhension des réalités changeantes et complexes du monde contemporain. Aussi modestement soit-il, ils pourraient aussi permettre d'enclencher un dialogue post-orientaliste et post-islamiste de type « cosmopolite » (Ernst \& Martin 2010), - et faire fi de ces terminologies qui ne sont pas satisfaisantes - dialogue qui pourrait contribuer de manière non hégémonique, ni introvertie, ni extravertie, au défi du renouvellement des études sur l'islam en Afrique et au-delà.

Centre d'études africaines, EHESS, Paris.

\section{BIBLIOGRAPHIE}

AmSELLE, J.-L.

1999 [1990] Logiques métisses. Anthropologie de l'identité en Afrique et ailleurs, Paris, Payot.

Arweck, E. \& Stringer, M. D. (eds.)

2002 Theorizing Faith. The Insider/Outsider Problem in the Study of Ritual, Birmingham, The University of Birmingham Press. 
AsAD, T.

1990 «Afterwords : From the History of Colonial Anthropology to the Anthropology of Western Hegemony », in G. Stocking (ed.), Colonial Situations, Madison, University of Wisconsin Press : 314-324.

AugÉ, M.

1994 Pour une anthropologie des mondes contemporains, Paris, ChampsFlammarion.

Ayi, B., Harrell, S. \& LunZy, M.

2007 Fieldwork Connections. The Fabric of Ethnographic Collaboration in China and America, Seattle, University of Washington Press.

BÂ, A. H.

2004 [1980] Vie et enseignement de Tierno Bokar, Le sage de Bandiagara, Paris, Éditions du Seuil.

BANK, A.

2008 «The "Intimate Politics" of Fieldwork: Monica Hunter and her African Assistants, Pondoland and the Eastern Cape, 1931-1932 », Journal of Southern African Studies, 34 (3) : 557-574.

BAZIN, J.

1996 «Interpréter ou décrire. Notes critiques sur la connaissance anthropologique », in J. REVEL \& N. WACHEL (dir.), Une école pour les sciences sociales. De la VI e section à l'École des hautes études en sciences sociales, Paris, Éditions du Cerf-EHESS.

Bensa, A.

2006 La fin de l'exotisme. Essais d'anthropologie critique, Paris, Anacharsis.

2007 «Empathie et objectivité : retour sur une expérience ethnographique de longue durée », Communication présentée aux « Débats de l'EHESS », Paris, 22-24 octobre.

Bensa, A. \& Fassin, D. (dir.)

2008 Les politiques de l'enquête. Épreuves ethnographiques, Paris, La Découverte.

BERLINER, D.

2008 « The Anthropologist in the Middle of a Tug-of-War», Men and Masculinities, 11 (2) : 174-185.

BIERSCHENK, T.

2007 Une anthropologie entre rigueur et engagement: essais autour de l'œuvre de Jean-Pierre Olivier de Sardan, Paris, Karthala.

Blowsnake, S., avec la contribution de Radin, P.

1920 The Autobiography of a Winnebago Indian, Berkeley, University of California Press (réimprimé en 1926 sous la direction de P. Radin, Crashing Thunder : the Autobiography of an American Indian, New York, Appleton \& Co. ; trad. en français sous le titre Autobiographie d'un indien Winnebago, Paris, Éditions Le Mail, 1989). 
Blundo, G. \& Olivier de Sardan, J.-P. (dir.)

2003 Pratiques de la description, Paris, Éditions de l'EHESS.

BoAs, F. \& Hunt, G.

1895 The Social Organization and the Secret Societies of the Kwakiutl Indians, Washington, D.C., Annual Report of the National Museum.

BotTe, R.

2000 «De l'esclavage et du daltonisme dans les sciences sociales », Journal des Africanistes, 70 (1-2) : 7-42.

Bousu, J.

2003 «La culture dogon: de l'ethnologie coloniale à l'anthropologie réciproque contemporaine », Clio en Afrique, 10, $<\mathrm{http} / / /$ sites.univ-provence.fr/wclio-af/d_fichiers10/culturedogon.html>.

Bousbina, S.

1992 «Musa Kamara, un savant "autodidacte" », Islam et Sociétés au sud du Sahara, 6: 75-81.

BRENNER, L.

1999 «The Study of Islam in Sub-Saharan Africa», ISIM Newsletter, $4: 31$.

Brettell, C. B. (ed.)

1993 When They Read What We Write: The Politics of Ethnography, Westport, Bergin \& Garvey.

Cefaï, D. (dir.)

2010 L'engagement ethnographique, Paris, Éditions de l'EHESS.

Cefaï, D. \& Amiraux, V.

1992 «Les risques du métier. Engagements problématiques en sciences sociales », Cultures \& Conflits, 47 (3) : 15-48.

Clifford, J.

1983a « On Ethnographic Authority », Representations, 1 : 118-146.

1983b " Power and Dialogue in Ethnography. Marcel Griaule's Initiation », in G. STоскING (ed.), Observers Observed : Essays on Ethnographic Fieldwork, Madison, The University of Wisconsin Press : 121-156.

Clifford, J. \& Marcus, G.

1986 Writing Culture, the Poetics and Politics of Ethnography, Berkeley, University of California Press.

Clough, P.

2006 «Knowledge in Passing: Reflexive Anthropology and Religious Awareness », Anthropological Quarterly, 79 (2) : 261-283.

Comte-Sponville, A.

2003 [1992] L'Amour, la solitude, Paris, Le Livre de Poche. 
D'Andrade, R. \& Scheper-Hughes, N.

1995 «Objectivity and Militancy: A Debate (1. Moral Models in Anthropology; 2. The Primacy of the Ethical) », Current Anthropology, 36 (3) : 399-440.

VAN DiJK, R. \& PELS, P.

1996 «Contested Authorities and the Politics of Perception: Deconstructing the Study of Religion in Africa », in T. RANGer \& R. WERBner (eds.), Postcolonial Identities in Africa, London-New York, Zed books : 245-270.

ERnst, C. \& Martin, C. M. (eds.)

2010 Rethinking Islamic Studies: From Post-Orientialism to Cosmopolitanism, Columbia, University of South Carolina Press.

FABIAN, J.

1983 Time and the Other: How Anthropology Makes Its Object, New York, Columbia University Press (traduction française: Comment l'anthropologie construit son objet, Toulouse, Anacharsis, 2006).

1990 «Presence and Representation: The Other and Anthropological Writing », Critical Inquiry, 16 (4) : 753-772.

2006 «The Other Revisited : Critical Afterthoughts », Anthropological Theory, 6 : $139-152$

FANCELLO, S.

2008 «Travailler sans affinité : l'ethnologue chez les convertis», Journal des anthropologues consacré à «L'empathie en anthropologie», 114-115: 65-90.

Field, L. avec Seidner, C., Lang, J., Cambra, R., Silva, F., Hailstone, V., Marshall, D., Marshall, B., Lara, C., George, M. Sr., \& le comité culturel de la tribu YORUK

2008a Abalone Tales: Collaborative Explorations of Sovereignty and Identity in Native California, Durham, Duke University Press.

FIELD, L.

2008b " "Side by Side, or Facing One Another" : Writing and Collaborative Ethnography in Comparative Perspective », Collaborative Anthropologies, 1 : 32-50.

Fletcher, A. C. \& La Flesche, F.

1911 The Omaha Tribe. Twenty-seventh Annual Report of the Bureau of American Ethnology, Washington, D.C., Government Printing Office.

Fluehr-Lobban, C. (ed.)

1990 Ethics and the Profession of Anthropology: Dialogue for a New Era, Philadelphia, University of Pennsylvania Press.

Fluehr-LobBan, C.

2008 «Collaborative Anthropology as Twenty-First Century Ethical Anthropology », Collaborative Anthropologies, 1, <http://www.marshall.edu/coll-anth/toc.html>.

FREITAG, U.

1997 « The Critique of Orientalism », in M. BentLey (ed.), Routledge Companion to Historiography, London-New York, Routledge : 620-638. 
GARFINKEL, H.

1967 Studies in Ethnomethodology, Englewood Cliffs, Prentice-Hall.

GeERTZ, C.

1973 «Thick Description: Toward an Interpretive Theory of Culture », in C. GeERTZ (ed.), The Interpretation of Cultures : Selected Essays, New York, Basic Books : 3-30 (trad. française : «La description dense. Vers une théorie interprétative de la culture, Enquête », Anthropologie, histoire, sociologie, $6: 73-105,1998)$.

1983 « From the Native's Point of View. On the Nature of Anthropological Understanding », in C. GEERTZ (ed.), Local Knowledge : Further Essays in Interpretive Anthropology, New York, Basic Books : 55-70.

GHASARIAn, C.

1997 «Les désarrois de l'ethnographe », L'Homme, 37 (143) : 189-198.

Griaule, M.

1948 Dieu d'eau, entretiens avec Ogotommêli, Paris, Librairie Arthème Fayart.

Gugelberger, G. M.

1996 The Real Thing : Testimonial Discourse and Latin America, Durham, Duke University Press.

HARRISON, C.

2003 [1988] France and Islam in West Africa, 1860-1960, Cambridge, Cambridge University Press.

Hillesum, E.

1995 [1985] Une vie bouleversée. Journal 1941-43, suivi de Lettres de Westerbork, Paris, Éditions du Seuil.

HodGKIN, E.

1990 «Islamism and Islamic Research in Africa », Islam et Sociétés au sud du Sahara, 4 : 73-130.

HounTONDJ, P.

1990 «Scientific Dependence in Africa Today », Research in African Literatures, 21 (3) : 5-15.

1995 « Producing Knowledge in Africa Today. The Second Bashorun M. K. O. Abiola Distinguished Lecture », African Studies Review, 38 (3) : 1-10.

JACKSON, M.

1998 Minima Ethnographica. Intersubjectivity and the Anthropological Project, Chicago, University of Chicago Press.

Jules-Rosette, B.

1976 «The Conversion Experience : the Apostles of John Maranke», Journal of Religion in Africa, 7 (2) : 132-164. 
KAMARA, M. S.

1998 Florilège au jardin de l'histoire des Noirs. Zuhûr al-Basâtîn. I : L'aristocratie peule et la révolution des clercs musulmans (Vallée du Sénégal), Traduction de Saïd Bousbina, introduite par J. Schmitz, avec la collaboration de C. Becker, A. B. Diop, C. Hames et al., Paris, Éditions du CNRS.

KANE, O,

2003 Intellectuels non-europhones, document de travail, Dakar, Codesria, $<$ www.codesria.org/IMG/pdf/ousmane_kane.pdf $>$.

KASSAM, T. R.

2004 «Balancing Acts : Negociating the Ethics of Scholarship and Identity », in J. I. Cabezon \& S. G. Davaney (eds.), Identity and the Politics of Scholarship in the Study of Religion, London-New York, Routledge : 133-161.

Kleinman, S. \& Copp, M. A.

1993 Emotions and Fieldwork, Newbury Park, Calif., Sage Publications.

Kramer, M.

2001 Ivory Towers on Sand: The Failure of Middle Eastern Studies in America, Washington, Washington Institute for Near East Policy.

LASSITER, L. A.

2005a «Collaborative Eyhnography and Public Anthropology », Current Anthropology, 46 (1) : 83-106.

2005b The Chicago Guide to Collaborative Ethnography, Chicago, University of Chicago Press.

Lassiter, L. A., Goodhall, H., Campbell, E., Johnson, M. N. et les étudiants DU SÉMinAIRE « OTHER Side OF MidDlETOWN »

2004 The Other Side of Middletown : Exploring Muncie's African American Community, Lanham, AltaMira Press.

LAUNAY, R.

2006 «An Invisible Religion? Anthropology's Avoidance of Islam in Africa», in M. Ntarangwi, D. Mills \& M. H. M. Babiker (eds.), African Anthropologies : History, Critique, and Practice, London, Zed Books ; Dakar, Codesria : 188-203.

Le Pichon, A.

1991 Le regard inégal, Paris, J. C. Lattès.

LESERVOISIER, O. \& VIDAL, L. (eds.)

2007 L'anthropologie face à ses objets : nouveaux contextes ethnologiques, Paris, Éditions des Archives contemporaines.

LuKENS-Bull, R. A.

2007 «Lost in a Sea of Subjectivity: The Subject Position of the Researcher in the Anthropology of Islam », Contemporary Islam : Dynamics of Muslim Life, 1 (2) : 173-192. 
Marcus, G.

1998 Ethnography Through Thick and Thin, Princeton, Princeton University Press.

Marcus, G. \& Fisher, M.

1986 Anthropology as Cultural Critique : An Experimental Moment in the Human Sciences, Chicago, The University of Chicago Press.

MARY, A.

2000 «L'anthropologie au risque des religions mondiales », Anthropologie et Sociétés, numéro spécial «Terrains d'avenir», 24 (1): 117-135.

MAUPOIL, B.

1943 La Géomancie à l'ancienne Côte des Esclaves, Paris, Institut d'ethnologie.

McBeth, S.

1993 «Myths of Objectivity and the Collaborative Process in Life History Research », in C. B. BRETTELL (ed.), When They Read What We Write: The Politics of Ethnography, Westport, Bergin \& Garvey : 145-162.

McCutcheon, R. T. (ed.)

1999 The Insider/Outsider Problem in the Study of Religion : A Reader, LondonNew York, Cassell.

McGrane, B.

1989 Beyond Anthropology, Society, and the Other, New York, Columbia University Press.

MiRAN, M.

2006 Islam, histoire et modernité en Côte d'Ivoire, Paris, Karthala.

Miran, M. \& El Hadj Vissoh, C. A.

2009 «(Auto)biographie d'une conversion à l'islam: Regards croisés sur une histoire de changement religieux dans le Bénin contemporain », Cahiers d'Études africaines, XLIX (3), 195 : 655-704.

MORIN, E.

2008 [1977] La méthode, vol. 2 : La vie de la vie, Paris, Éditions du Seuil.

Mosse, D.

2006 «Anti-Social Anthropology? Objectivity, Objection, and the Ethnography of Public Policy and Professional Communities », Journal of the Royal Anthropological Institute, 12 : 935-956.

Mudimbe, V.

1988 The Invention of Africa: Gnosis, Philosophy, and the Order of Knowledge, Bloomington-Indianapolis, Indiana University Press.

Овво, С.

1990 «Adventures with Fieldnotes », in R. SANJEK (ed.), Fieldnotes : The Makings of Anthropology, Ithaca, Cornell University Press. 
Okely, J. \& Callaway, E. (eds.)

1992 Anthropology and Autobiography, London-New York, Routledge.

OLIVIER DE SARDAN, J.-P.

1988 «Jeu de la croyance et "je" ethnologique : exotisme religieux et ethnoégocentrisme », Cahiers d'Études africaines, XXVIII (3-4), 111-112: 527540.

1989 «Le réel des autres », Cahiers d'Études africaines, XXIX (1), 113 : 127-135.

1998 «Émique», L'Homme, 147 : 151-166.

2008 La rigueur du qualitatif: Les contraintes empiriques de l'interprétation socio-anthropologique, Louvain-la-neuve, Academia-Bruylant.

Owusu, M.

1976 «Colonial and Postcolonial Anthropology of Africa: Scholarship or Sentiment? », in A. ARENS (ed.), A Century of Change in Eastern Africa, The Hague, Mouton.

Piault, C.

1975 Prophétisme et thérapeutique: Albert Atcho et la communauté de Bregbo, Paris, Hermann.

RANCO, D. J.

2006 «Toward a Native Anthropology : Hermeneutics, Hunting Stories, and Theorizing from Within », Wicazo Sa Review, 21 (2) : 61-78.

RAPPAPORT, J.

2008 «Beyond Participant Observation : Collaborative Ethnography as Theoretical Innovation », Collaborative Anthropologies, 1: 1-31.

REYNOLDS, J.

2002 «Mining Information and Manufacturing Scholarship. Are Western-Based Researchers Just Another Extractive Economy? », Communication présentée au West African Research Association Interdisciplinary Symposium on Fieldwork, Africa, Dakar.

SALVAING, B.

2003b « Un exemple d'autobiographie à deux voix venue d'Afrique : les mémoires d'Almamy Maliki Yattara », Mots pluriels, 23, $<$ http://motspluriels.arts.uwa.edu.au/MP2303bs.html>.

SAlvaing, B. \& AL-HADJi BALdÉ, T. M.

2008 Une vie au Fouta Djalon, Brinon-sur-Sauldre, Grandvaux.

Salvaing, B. \& Yattara, A. M.

2000 Almamy. Une jeunesse sur les rives du fleuve Niger, Brinon-sur-Sauldre, Grandvaux.

2003a Almamy, l'âge d'homme d'un lettré malien, Brinon-sur-Sauldre, Grandvaux. 
SANJEK, R.

1993 «Anthropology's Hidden Colonialism : Assistants and Their Ethnographers », Anthropology Today, 9 (2) : 13-18.

SCHUMAKER, L.

2001 Africanizing Anthropology: Fieldwork, Networks, and the Making of Cultural Knowledge in Central Africa, Durham, Duke University Press.

SHOSTAK, M.

2000 Nisa: The Life and Words of a Kung Woman, Cambridge, Harvard University Press.

SмITH, M.

1954 Baba of Karo. A Woman of the Muslim Hausa, London, Faber (réédité en 1981 par Yale University Press, New Haven et paru en français en 1969 sous le titre Baba de Karo. L'autobiographie d'une musulmane haoussa du Nigeria. Textes de Baba Giwa, Paris, Plon).

SouTy, J.

2007 Pierre Fatumbi Verger. Du regard détaché à la connaissance initiatique, Paris, Maisonneuve et Larose.

SPENCER, J.

1989 «Anthropology as a Kind of Writing », Man, 24 : 145-164.

TRIAUd, J.-L.

2009 « D'un maître à l'autre : l'histoire d'un transfert. Amadou Hampaté Bâ entre Tierno Bokar et Théodore Monod (1938-1954)», Sociétés politiques comparées. Revue européenne d'analyse des sociétés politiques, 20 :

$<$ http://www.fasopo.org/reasopo/n20/article.pdf>.

2010 «L'islam au sud du Sahara. Une saison orientaliste en Afrique occidentale. Constitution d'un champ scientifique, héritages et transmissions », Cahiers d'Études africaines, L (2-4), 198-199-200 : 907-950.

VANBEEK, W.

1991 « Dogon Restudied, A Field Evaluation of the Work of Marcel Griaule », Current Anthropology, 32 (2) : 139-167.

Van MaAnen, J.

1988 Tales of the Field: On Writing Ethnography, Chicago, Chicago University Press.

Ville, J.-L. \& GuYo, A.

2004 Le dernier éléphant, Histoire d'un chasseur kenyan, Paris, Autrepart.

WOLF, M.

1992 A Thrice Told Tale : Feminism, Postmodernism \& Ethnographic Responsibility, San Francisco, Standford University Press. 


\section{RÉSUMÉ}

Cet article revient sur une expérience de co-écriture entre ethnologue et imam, dont le fruit est une longue étude parue dans les Cahiers d'Études africaines en 2009. Monologue réflexif du seul chercheur, I'article s'inscrit dans le genre des récits anthropologiques à la première personne, en privilégiant une posture (auto)critique contre l'écueil des excès narcissiques. II retrace la généalogie de cette démarche dialogique, la rencontre sur le terrain béninois avec I'imam d'Allada El Hadj Akan Charif Vissoh, la réception de son récit de conversion à l'islam et les étapes postterrain de la mise en écriture, refaçonnée à deux. Dans le même temps, il questionne les atouts et les limites de cette coproduction intellectuelle qui, en provoquant la poursuite du travail de terrain dans l'écriture et le renvoi du travail d'écriture vers l'interlocuteur sur le terrain, brouille les cartes entre interprète et interprété, outsider et insider, ethnologue et acteur religieux. L'article prend par ailleurs prétexte de l'exposé de cette aventure singulière pour esquisser un voyage de modeste encablure dans l'histoire, l'épistémologie et la méthodologie d'un courant ancien et longtemps marginalisé au sein de la discipline mais désormais en plein renouveau, celui de l'anthropologie collaborative.

\section{ABSTRACT}

When Ethnologist and Imam Become Co-authors. A journey into Collaborative Anthropology. - This article comes back on a co-writing experience between ethnologist and imam, which produced a study published in the Cahiers d'Études africaines in 2009. Reflexive monologue from the researcher's point of view, the article comes within the genre of the anthropological tale in first person, against which excesses an (auto)critical posture is attempted. It narrates the genealogy of this dialogical approach, the encounter in the field in Benin with Allada's imam El Hadj Akan Charif Vissoh, the reception of his conversion's story, and the post-field stages of the putting-into-writing, jointly reworked by both co-authors in-the-making. At the same time, it questions the strengths and weaknesses of this intellectual coproduction, which provokes the pursuit of fieldwork into deskwork and the sharing of the writing process with the field partner, thus clouding the traditional roles between interpreter and interpreted, outsider and insider, ethnologist and religious actor. Furthermore, the article takes occasion of the account of this singular adventure to engage in a modest journey into the history, epistemology, and methodology of an old and long marginalised trend within the discipline yet now revived : that of collaborative anthropology.

Mots-clés/Keywords : Bénin, Côte-d'Ivoire, anthropologie collaborative, épistémologie, méthodologie/Benin, Côte-d'Ivoire, collaborative anthropology, epistemology, methodology. 